\title{
Análise da Revisão Cochrane: Efectividade de Intervenções Educacionais para Pacientes sobre Medicamentos Sujeitos ou não a Receita Médica. Cochrane Database Syst Rev. 2013;4:CD008416.
}

\author{
Analysis of the Cochrane Review: Multimedia Educational Interventions for \\ Consumers about Prescribed and Over-the-counter Medications. Cochrane \\ Database of Systematic Reviews 2013;4:CD008416.
}

António VAZ CARNEIRO ${ }^{1,2}$, João COSTA $1,2,3,4$

Acta Med Port 2014 Jan-Feb;27(1):4-5

Palavras-chave: Educação de Doentes; Medicamentos sem Prescrição; Multimédia; Revisão Sistémica.

Keywords: Multimedia; Nonprescription Drugs; Patient Education as Topic; Systematic Reviews.

\section{RESUMO}

Para um correcto e racional uso dos medicamentos por parte dos pacientes é necessária uma compreensão detalhada da informação disponível. A informação terapêutica deve ser disponibilizada de tal maneira que os pacientes consigam compreender todos os aspectos inerentes ao esquema de tratamento que lhes foi prescrito (ou que tomam por decisão própria). Nesta revisão sistemática, os autores analisaram um conjunto de estudos sobre a efectividade de intervenções educacionais multimédia sobre medicamentos sujeitos ou não a receita médica, em pacientes de todos os grupos etários, concluindo que a intervenção educacional multimédia é mais eficaz que as informações dadas pelos profissionais de saúde responsáveis pela assistência aos doentes, ou que a ausência de informação.

\section{ABSTRACT}

Understanding of the relevant information is especially important in the area of drug treatment, to guarantee an appropriate and rational use of medications by patients. The relevant information must be delivered in a way that patients understand all aspects of the treatment regimen they are taking. In this systematic review the authors analyzed a set of studies on the effectiveness of multimedia educational interventions about medications (prescribed or not) in patients of all ages, concluding that the aforementioned interventions are more effective than usual care (non-standardized education provided by health professionals as part of usual clinical care) or no education.

\section{QUESTÃO CLÍNICA}

Qual é a efectividade das intervenções educacionais sobre medicamentos realizadas pelos profissionais de saúde (médicos em particular) no aumento do conhecimento, técnicas de utilização e adesão terapêutica?

\section{OBJECTIVOS}

Esta revisão sistemática procurou avaliar o impacto de um conjunto de intervenções multimédia sobre informação farmacológica na utilização de medicamentos em pacientes de todos os grupos etários.

\section{METODOLOGIA}

Foram pesquisadas várias bases de dados - Cochrane Central Register of Controlled Trials (CENTRAL, The Cochrane Library 2011, Issue 6), MEDLINE (1950 to June 2011), EMBASE (1974 to June 2011), CINAHL (1982 to June 2011), PsycINFO (1967 to June 2011), ERIC (1966 to June 2011), ProQuest Dissertation \& Theses Database (to June 2011) e listas de referências de artigos publicados - para identificação de ensaios clínicos aleatorizados, prospectivos e controlados (Randomized Clinical Trials RCTs), ou quasi-RCTs, que avaliaram técnicas educacionais multimédia incidindo sobre medicamentos (prescritos individualmente ou não pelo médico) em pacientes de todos os grupos etários (incluindo crianças). A extracção dos dados e sua análise em termos de risco de vieses foi feita por dois investigadores que contactaram, quando necessário, os autores dos estudos para informação adicional.

\section{RESULTADOS}

Foram incluídos 24 estudos (com um total de 8112 participantes) de qualidade metodológica variável e com vieses potenciais, sem indicação de diferenças entre os outcomes utilizados e com heterogeneidade marcada (nos comparadores e outcomes).

As intervenções educacionais multimédia sobre medicamentos, quando comparadas com as informações dadas pelos profissionais de saúde responsáveis pela assistência aos doentes ou com a ausência pura e simples de informação, estiveram associadas a:

1. Centro de Estudos de Medicina Baseada na Evidência. Faculdade de Medicina. Universidade de Lisboa. Lisboa. Portugal.

2. Centro Colaborador Português da Rede Cochrane Iberoamericana. Lisboa. Portugal.

3. Unidade de Farmacologia Clínica. Instituto de Medicina Molecular. Faculdade de Medicina. Universidade de Lisboa. Lisboa. Portugal.

4. Laboratório de Farmacologia Clínica e Terapêutica. Faculdade de Medicina da Universidade de Lisboa. Lisboa. Portugal.

Recebido: 20 de Janeiro de 2014 - Aceite: 21 de Janeiro de 2014 | Copyright @ Ordem dos Médicos 2013 
1) melhoria do conhecimento adquirido (Standardised Mean Difference $[S M D]=1,04 ;$ IC $95 \%$ : 0,49 a 1,58; seis estudos com 817 participantes);

2) melhoria em termos de aquisição de competências (por exemplo, na utilização de nebulizador para asma em que se verificou uma diferença média de 18,32\%; IC 95\%: 11,92 a 24,73; 2 estudos com 94 participantes).

Não se verificaram diferenças significativas em termos de adesão terapêutica (Risco Relativo [RR] de adesão = 1,02; IC 95\%: 0,96 a 1,08; 2 estudos com 4552 participantes).

\section{CONCLUSÕES}

Estes resultados indicam que, quando comparado com as informações dadas pelos profissionais de saúde responsáveis pela assistência aos doentes ou com a ausência pura e simples de qualquer informação, um conjunto de intervenções educacionais multimédia sobre utilização de medicamentos (para todos os grupos etários) é superior em termos de conhecimento adquirido e aquisição de competências pelos pacientes, sem no entanto existir um impacto na adesão terapêutica.

\section{COMENTÁRIO}

A iliteracia em saúde pode definir-se - segunda a OMS - como ..."estando ligada ao conceito de literacia em geral, implicando o conhecimento, motivação e competências para o acesso, compreensão, avaliação e aplicação da informação em saúde que sirva de base aos julgamentos e tomada de decisão quotidianas no que concerne os cuidados de saúde, prevenção de doença e promoção de saúde na manutenção e melhoria da qualidade de vida."*

A literacia em saúde é presentemente mais importante que nunca, já que nas cada vez mais complexas sociedades modernas uma significativa percentagem das mensagens em saúde que chegam ao cidadão médio são confusas e de baixa qualidade, fazendo com que aquele seja desinformado e fique susceptível a mitos e crenças de todos os géneros. Existe de resto evidência científica de boa qualidade demonstrando que a um menor grau de literacia em saúde dos pacientes se associa uma maior taxa de internamento e de utilização de serviços de urgência, menor adesão terapêutica, deficiente compreensão de resultados de exames auxiliares de diagnóstico e, nos idosos, maior

\footnotetext{
* WHO. Health Literacy. The solid facts. 2013.
}

\section{REFERÊNCIAS}

1. Berkman ND, Sheridan SL, Donahue KE, Halpern DJ, Crotty K. Low health literacy and health outcomes: an updated systematic review. Ann Int Med. 2011;155:97-107.

2. Ley P. Satisfaction, compliance and communication. Br J Clin Psychol.

morbilidade e até mortalidade. ${ }^{1}$ A diminuição desta situação parece portanto óbvia e necessária, a questão é como consegui-lo.

Na presente revisão sistemática os autores procuraram determinar o eventual benefício de intervenções especificamente dirigidas a aumentar nos pacientes o conhecimento sobre medicação, através da análise de estudos de base experimental (RCTs ou quais-RCTs) - os quais fornecem a melhor prova de causalidade entre uma intervenção e um resultado - comparando-as com a prática habitual. As intervenções - designadas como multimédia - incluíram textos, diagramas, imagens, áudios, animações e vídeos. Os resultados indicam - nestes 24 estudos de modesta qualidade (por exemplo, uma percentagem significativa dos estudos não detalhava as intervenções educacionais) - que as intervenções multimédia, como um todo, são mais eficazes que as práticas de rotina na melhoria do conhecimento sobre fármacos e na aquisição de desempenhos práticos (por exemplo uso de nebulizador para a asma), mas infelizmente não parecem ter um efeito sobre a adesão terapêutica.

Estes resultados são importantes, já que se sabe que a retenção da informação dada por médicos aos doentes é muito deficiente: $40-80 \%$ desaparece imediatamente ${ }^{2}$ e a quantidade de informação retida é inversamente proporcional à apresentada. ${ }^{3}$ Dado que a informação sobre a utilização de fármacos disponibilizada por metodologias multimédia é superior à fornecida por os médicos e outros profissionais de saúde, então a combinação de ambos deverá ter efeitos maximizados e de dimensão importante.

\section{IMPLICAÇÕES PARA PRÁTICA}

- Os doentes/pacientes necessitam de ser informados sobre a utilização de fármacos, não só no que diz respeito ao benefício esperado como ao seu risco potencial

- Esta necessidade cresce com a complexidade dos esquemas terapêuticos

- A adição de intervenções educacionais multimédia ao aconselhamento clínico aumenta significativamente o conhecimento e desenvolve as aptidões práticas dos doentes

- O aumento de adesão terapêutica necessita de outras abordagens, já que as referidas não parecem ter benefícios mensuráveis.
1982;21:241-54.

3. Kessels RP. Patients' memory for medical information. J Royal Soc Med. 2003;96:219-22.

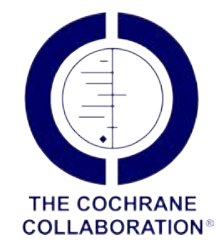




\section{Análise da Revisão Cochrane: Efectividade de} Intervenções Educacionais para Pacientes sobre Medicamentos Sujeitos ou não a Receita Médica. Cochrane Database Syst Rev. 2013;4:CD008416. Acta Med Port 2014:27:4-5

Publicado pela Acta Médica Portuguesa, a Revista Científica da Ordem dos Médicos

Av. Almirante Gago Coutinho, 151

1749-084 Lisboa, Portugal.

Tel: +351 218428215

E-mail: submissao@actamedicaportuguesa.com

www.actamedicaportuguesa.com

ISSN:0870-399X | e-ISSN: 1646-0758

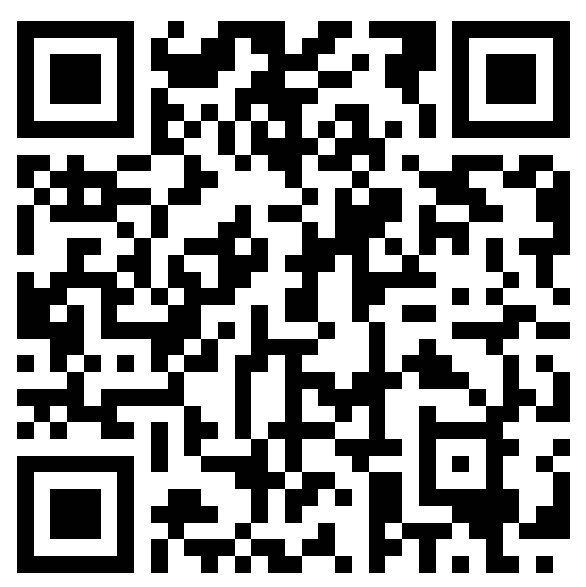

